\title{
Recurrent retinoblastoma with intracranial extension post enucleation
}

\author{
Pulkit Maru $^{1}$, Ankush Sarwal ${ }^{2 *}$, Sweety Thakker ${ }^{3}$, Neetika sarwal ${ }^{4}$, Anant Pore ${ }^{5}$ \\ ${ }^{\mathbf{1 , 2 , 4 , 5}}$ Fellow, ${ }^{\mathbf{3}}$ Resident, ${ }^{\mathbf{1}}$ Dept. of Surgical oncology, ${ }^{\mathbf{1 , 2 , 4 , 5}}$ Tata Medical Centre, Kolkata, ${ }^{\mathbf{3}}$ B.J. Medical college, Civil Hospital Ahmedabad, \\ Gujarat India
}

\section{*Corresponding Author: Ankush Sarwal}

Email: drankushsarwal@yahoo.in

\begin{abstract}
Retinoblastoma amounts to be the most common intraocular childhood malignancy. More than 95\% retinoblastoma are diagnosed before age five. Retinoblastoma may be sporadic or inherited. In both cases the tumor origin is biallelic mutation. Staging of retinoblastoma is based on intraocular location, extraocular \& extraorbital spread, central nervous system involvement and systemic metastases. Diagnosis and management of retinoblastoma demands multidisciplinary approach, for which accurate imaging is indispensable. Concerns of the effects of radiation in retinoblastoma have led to a shift from radiation therapy to chemotherapy and locoregional treatment and magnetic resonance imaging becoming the standard imaging modality for diagnosis, staging and treatment monitoring.
\end{abstract}

Keywords: Retinoblastoma, Retinoblastoma with Intracranial extension, Recurrent retinoblastoma.

\section{Introduction}

Retinoblastoma is the commonest intraocular tumor of childhood (usually presenting below age 5 years) and is thought to be congenital in origin. ${ }^{4}$ It is highly malignant tumor with an incidence of 1 case of retinoblastoma per 18,000-30,000 live births, varying on the demographics. ${ }^{3}$ It arises from the neuroectodermal cells of the retina and must be differentiated from other benign lesions for early management and better prognosis. Clinically, leucocoria is associated with proptosis, which may be bilateral. ${ }^{4}$ The main patterns of the lesion are endophytic, exophytic or diffuse type. ${ }^{3}$ The intraocular mass with calcification and areas of necrosis, associated retinal detachments and vitreous involvement (endophytic type) can be well visualized on Ultrasonography (USG). Magnetic Resonance Imaging (MRI) is very useful in determining retrobulbar and extraocular spread, intracranial involvement, and exclude trilateral retinoblastoma with pineal gland tumors when bilateral disease is detected. On MR, they are hyperintense to normal vitreous on T1WI, moderate to markedly hypointense on T2WI depending on the calcification, demonstrated well with contrast administration and thin sections $(1.5-3 \mathrm{~mm})$ with fat-suppression. It is important to differentiate the tumor from persistent hyperplastic primary vitreous (PHPV), retinopathies of prematurity (ROP), Coat's disease (primary retinal telengiectases), organized subretinal hemorrhage, endophthalmitis, and other intraocular masses. ${ }^{4}$

\section{Case Report}

A 3-year-old female child presented with operative history of enucleation of right eye for retinoblastoma 6 months back. Presently her parents give complain of swelling in right eye of the child noticed in the last 1 month, which is bulging out of right orbital margins. There is no history of discharge and no history of fever. Patient had received preoperative chemotherapy. Postoperative biopsy revealed retinoblastoma and immediate postoperative MRI revealed no residual lesion following enucleation.

\section{Imaging Findings}

MRI plain and postcontrast study was done which revealed a large heterogeneously enhancing altered signal intensity intraorbital mass lesion on right side which filled the entire orbit and was protruding anteriorly well beyond transzygomatic line. The lesion appears hypointense on T1WI and isointense on T2WI with few hypointense areas which shows blooming on FFE images suggestive of calcified areas. The lesion showed restriction on diffusion weighted images. Visualized anterior most part of right optic nerve was seen to be encased by mass lesion and posterior part of intraorbital segment of optic nerve appear thickened with peripheral enhancement. Strip of fluid signal intensity collection was noted in anterior aspect of the lesion.

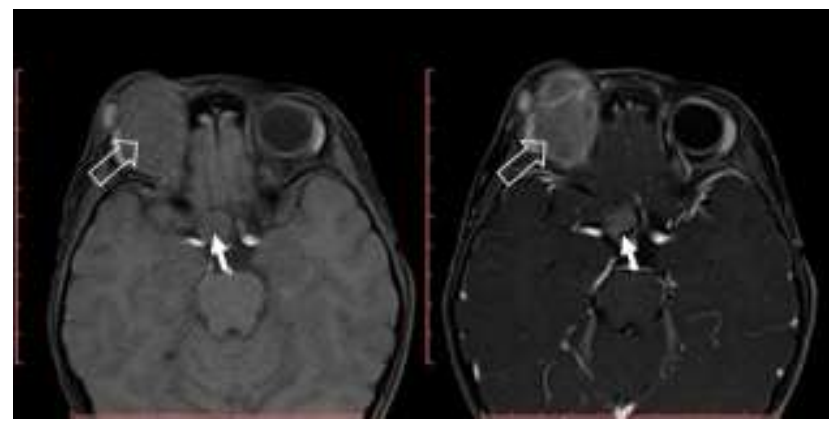

Fig. 1: T1 FAT-SAT precontrast and postcontrast images shows intraorbital mass (open arrow) with intracranial extension (solid arrow)

The lesion showed intracranial extension as evidenced by a similar characteristic nodular enhancing mass lesion involving cisternal segment of optic nerve and optic chiasm. This intracranial component abuts right straight gyrus, medial aspect off right basifrontal lobe, infundibular stalk, clinoid and supraclinoid (C5, C6) segments of right ICA. Pineal region shows no mass lesion. Left eyeball was normal. 


\section{Discussion}

MRI is the preferred imaging method in the evaluation of an intraocular lesion in a pediatric patient because there is no radiation exposure. ${ }^{2}$ In addition, MRI is superior to computed tomography (CT) in providing anatomical detail. As strong evidence has come forward in correlating sarcoma with radiation exposure in patients with retinoblastoma, radiotherapy and CT scan are now contraindicated in diagnosed and suspected patients of retinoblastoma. ${ }^{2}$ The child in the case vignette underwent MR examination without and with Gadolinium enhancement focusing on the brain and orbits. The sequences through the orbit were obtained as high-resolution images using a $3 \mathrm{~mm}$ slice thickness and a field of view as small as possible to accommodate both orbits. Such a protocol facilitates detection of small satellite lesions in the involved eye (referred to as vitreous or subretinal seedings), discovery of small contralateral retinoblastomas, and visualization of extraocular extension and optic nerve involvement. Additional images through the brain were obtained to exclude leptomeningeal spread as well as trilateral retinoblastoma (bilateral retinoblastoma with pineoblastoma) or quadrilateral retinoblastoma (trilateral retinoblastoma with pituitary tumour). The growth pattern of retinoblastoma can be divided into endophytic (growing into the globe), exophytic (growing toward the sclera), or diffuse infiltrating. The T2-weighted images best demarcate endophytic growth as they emphasize the contrast between the very bright vitreous and aqueous humor from the dark appearing intraocular lesion. Intraocular lesions are usually markedly less conspicuous on the T1-weighted images, in particular when small in size. In contrast, the Gadoliniumenhanced, fat-suppressed images are superior in detection of en plaque, exophytic and diffuse infiltrating retinoblastoma by accentuating the dark appearance of the collagen fibers within the sclera and suppressing the bright signal of the intraorbital fat. Different imaging planes, preferentially in axial and coronal direction, are required to assess the entire circumference of the globe and to detect a small intraocular lesion or exophytic growth. Both imaging planes are also helpful in mapping optic nerve infiltration by tumor.

MR susceptibility weighted images must be taken to demonstrate intratumoral calcification which can also be confirmed on ultrasonography as hyperechoic foci. Some cases of retinoblastoma may lack significant calcification or the calcification may be too small to be detected on the $3 \mathrm{~mm}$ slice thick images. Therefore, ocular ultrasound is particularly useful to detect a small focus of intralesional calcification.

MRI plays a significant role in staging the disease based on the commonly used International Classification of Retinoblastoma ${ }^{5}$ (ICRB)

Group A: Small intraretinal tumors $(<3 \mathrm{~mm})$ away from foveola and disc.

Group B: Tumors > 3mm, macular or juxtapapillary location, or with subretinal fluid.
Group C: Tumor with focal subretinal or vitreous seeding within $3 \mathrm{~mm}$ of tumor.

Group D: Tumor with diffuse subretinal or vitreous seeding $>3 \mathrm{~mm}$ from tumor.

Group E: Extensive retinoblastoma occupying $>50 \%$ of the globe with or without neovascular glaucoma, hemorrhage, extension of tumor to optic nerve or anterior chamber.

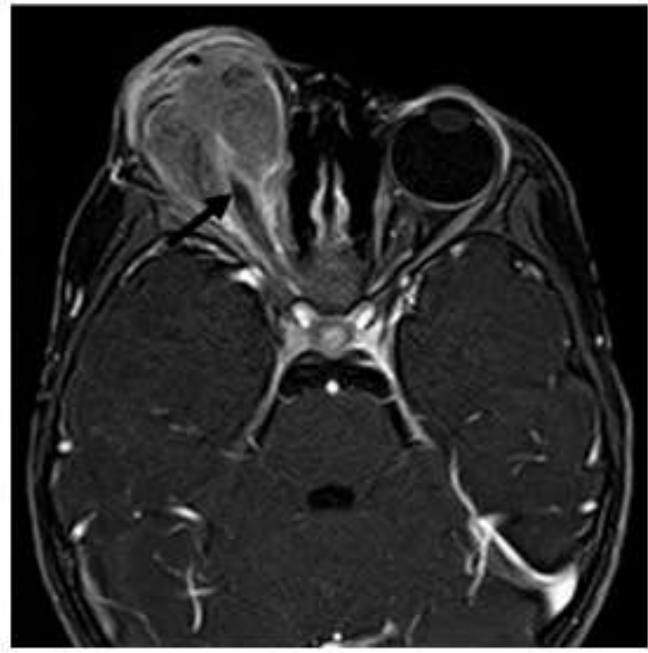

Fig. 2: post contrast T1 FAT-SAT image shows encasement of optic nerve stump by mass and enhancement of right optic nerve.

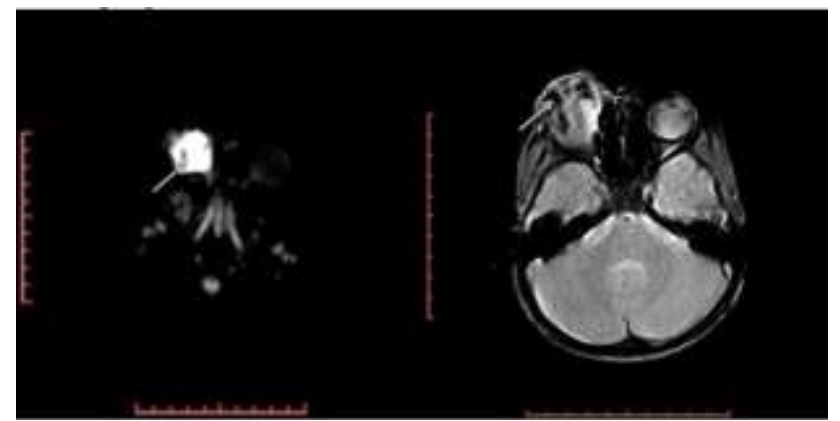

Fig. 3: DWI (b1000) image on the left shows that the lesion shows diffusion restriction and the FFE image on the right shows multiple dense foci of blooming within the lesion suggestive of calcification.

Specific associated findings or certain sites of involvement by the retinoblastoma are important for staging purposes. Some of the spread might be subtle and overlooked or interpreted as not important. Critical factors that might lead to significant change in tumor staging include vitreous hemorrhage or seeding, iris neovascularization, involvement of sclera or extrascleral tissue, optic nerve invasion and CNS involvement.

Vitreous hemorrhage presents as high signal on the T1weighted images or as a fluid hematocrit level on the axial T2 images within the vitreous body. Presence of vitreous hemorrhage automatically upstages the retinoblastoma into Group E tumor. ${ }^{2}$ Vitreous seeding primarily occurs with an endophytic retinoblastoma. The vitreous seeds are initially 
detected on ophthalmoscopy and first become visible on imaging when 1 or $2 \mathrm{~mm}$ in size. High-resolution T2weighted images are the best sequence for their detection. Presence of vitreous seeding upstages the retinoblastoma to Group C or D, depending on the extent of dissemination. ${ }^{2}$ Neovascularization of the iris is thought to be related to retinal and tumoral tissue hypoxia, resulting in reactive vessel proliferation. Clinically, iris neovascularization or 'rubeosis iridis' is seen as fine blood vessels on the surface of the iris or within the anterior chamber angle. Radiologically, it manifests as enhancement of the anterior chamber on Gadolinium-enhanced T1-weighted images. Neovascularization of the iris is not included in the ICRB as it is currently unclear if it is simply reactive in nature or a marker of progressive disease. The sclera is represented as a thin black line on MR images, interposed between the enhancing choroid and the intraorbital fat. ${ }^{2}$ Disruption of the dark line by an enhancing tumor is consistent with scleral involvement and a Group E tumor. The progressive growth of retinoblastoma through the sclera into the intraorbital fat (extrascleral spread) stages the patient to Group F. ${ }^{2}$ Optic nerve invasion is defined as retinoblastoma infiltration into the optic nerve beyond the lamina cribrosa. In general, optic nerve involvement is associated with poor prognosis with increasing mortality rates observed with progressive extent of optic nerve infiltration. It is best detected on highresolution contrast-enhanced T1-weighted images with fatsuppression in coronal and axial planes. It manifests as focal area of optic nerve enhancement that may be accompanied by optic nerve enlargement. ${ }^{2}$ CNS involvement can manifest as leptomeningeal spread or as trilateral or quadrilateral retinoblastoma. Leptomeningeal spread and suprasellar involvement is best visualized on Gadolinium-enhanced T1weighted images. In contrast, evaluation of enhancement of the pineal gland is not helpful because the normal pineal gland demonstrates marked physiological enhancement.
Therefore, involvement of the pineal gland is best visualized by increased volume and associated mass effect upon structures in the surroundings of the pineal gland. There is usually a median delay of 21 months from the time of the initial diagnosis of the retinoblastoma to identification of pineal or suprasellar involvement. Therefore, each patient with retinoblastoma should undergo MRI examination of brain at the time of staging as well as during each follow-up examination. $^{2}$

\section{Conflict of Interest: None.}

\section{References}

1. De Graaf P, Göricke S, Rodjan F. Guidelines for imaging retinoblastoma: imaging principles and MRI standardization. Pediatric Radiol 2012;42(1):2-14 doi:10.1007/s00247-011-2201-5.

2. Andreas M. Rauschecker, Chirag V. Patel, Kristen W. Yeom, Christina A. Eisenhut, Rakhee S. Gawande. Ebrahimi, and Heike E. Daldrup-Link. High-Resolution MR Imaging of the Orbit in Patients with Retinoblastoma: Radio Graphics 2012 32:5:1307-26.

3. Soni H C, Patel S B, Goswami K G, Gohil Y. Pictorial essay: USG of retinoblastoma. Indian J Radiol Imaging 2006;16:65768.

4. Hande PC, Talwar I. Multimodality imaging of the orbit. Indian J Radiol Imaging 2012;22:227-39

5. Carol L. The International Classification of Retinoblastoma Predicts Chemoreduction Success: Shields, Ophthalmol 113(12): $2276-80$.

6. Chantada G, Doz F, Antoneli CBG. A proposal for an international retinoblastoma staging system. Pediatr Blood Cancer 2006;47(6):801-5.

How to cite this article: Maru $\mathrm{P}$, Sarwal A, Thakker S, sarwal N, Pore A. Recurrent retinoblastoma with intracranial extension post enucleation. J Nutr, Metab Health Sci, 2019;2(2):68-70. 